\title{
A bridge too far? Attempting to bridge the treatment gap in osteoporosis
}

\author{
Bart L. Clarke ${ }^{1} \cdot$ Dolores M. Shoback $\mathbb{1}^{2}$ \\ Keywords Osteoporosis $\cdot$ Fracture $\cdot$ Treatment gap $\cdot$ BMD $\cdot$ DXA $\cdot$ FRAX
}

Received: 26 April 2020 / Accepted: 7 May 2020 / Published online: 21 May 2020

(c) Springer Science+Business Media, LLC, part of Springer Nature 2020

Despite substantial progress in the diagnosis and treatment of osteoporosis over the 25 years since the approval of the first bisphosphonate alendronate in 1995, osteoporosis remains significantly underrecognized and undertreated [1]. Osteoporosis causes low-trauma fractures that result in substantial morbidity, mortality, health care expense, and other societal costs. These consequences from fractures have persisted, despite major advances in the ability to predict future fractures based on bone mineral density (BMD) testing and a wide range of cost-effective therapeutic options approved by the FDA, EMA, and other regulatory agencies around the world [2]. Bisphosphonates were first developed beginning about 50 years ago, BMD by dual-energy x-ray absorptiometry (DXA) about 35 years ago, the first anabolic agent 18 years ago, and countryspecific FRAX algorithms to predict fractures in patients with osteopenia beginning 11 years ago.

The recent US National Osteoporosis Foundation report [3] describes how fractures affect the health of the US Medicare population, and recommends strategies that will reduce the risk of future fractures especially those occurring after a first fracture. This report shows that 2 million Americans 65 years and older sustained a total of 2.3 million fractures in 2015. Hospitalizations after these fractures exceeded combined hospitalizations for breast cancer, heart disease, and stroke. Among the patients with fractures, only $9 \%$ underwent BMD testing within the next 6 months, and in the first

Dolores M. Shoback

dolores.shoback@ucsf.edu

1 Mayo Clinic, 200 1st Street SW, Rochester, MN 55905, USA

2 San Francisco, Endocrine Research Unit-111N, San Francisco Department of Veterans Affairs Medical Center, University of California, 1700 Owens Street, 3rd Floor, Room 369,

San Francisco, CA 94158, USA
3 years following their fracture, 307,000 patients had their second fracture. The occurrence of these second fractures led to an additional expense estimated at US $\$ 6.3$ billion. Assuming that pharmacologic intervention after the first fracture would have reduced second fractures by even $20 \%$ leads to a potential savings estimate of US $\$ 1$ billion over 3 years after the first fracture. One category of osteoporosis medications, the bisphosphonates, is known to reduce future fractures by $40-70 \%$.

This report does not enumerate Medicare treatment rates for osteoporosis, but other recent reports have emphasized how treatment rates for secondary fracture prevention after a first fracture remain low, even in those at highest risk of a second fracture, such as those with incident hip or vertebral fractures $[4,5]$. Studies have shown that risk of a second fracture is significantly increased for about 2 years after an initial fracture. Treatment rates in the United States after an initial fracture are very low and reported to be declining. One study used a commercial insurance claims that database including 97,169 participants with incident hip fractures and mean age of 80.2 years (6), and showed that only $9.8 \%$ of the patients were given medication for osteoporosis in 2004, and only $3.3 \%$ in 2015 . In the group receiving treatment, the rate of subsequent nonvertebral fractures was significantly lower than in the untreated patients [6].

These low and declining treatment rates are most likely due to several factors. Concern about rare but serious side effects, such as jaw osteonecrosis or atypical femoral fractures, has caused some patients or their physicians to be reluctant to start treatment even in those at highest risk of fracture [5]. The benefit-risk ratio for most of these patients clearly favors treatment. Osteoporosis care is provided by a variety of specialties, each with their own guidelines and ways of managing care. The proliferation of nonaligned national and international guidelines has led to varying degrees of confusion in the medical community about when and who to treat. 
Significant variation exists within and between countries regarding recommendations for treatment of low-trauma fractures. Treatment rates in the UK and other countries are generally higher than in the United States. For example, the UK Clinical Research Database showed that about $50 \%$ of patients with incident hip fracture were treated in 2013 [7]. The variation in treatment rates within and between countries may be due to variation in models of care available to treat those with hip fracture. Multidisciplinary care provided by fracture liaison services has been shown to increase treatment rates and adherence to treatment, while reducing the risk of subsequent fractures [8]. Fracture liaison services provide an organized and cost-effective way of identifying and treating patients, and making sure that patients with hip fracture are referred back to their primary providers for ongoing care. Artificial intelligence may increase the detection rate of patients at increased risk of fracture more than fracture liaison clinicians by using natural language processing of $\mathrm{x}$-ray reports in the emergency department [9].

A number of initiatives around the world have been developed to promote availability of fracture liaison services, including the International Osteoporosis Foundation's Capture the Fracture initiative, and the secondary fracture prevention initiative sponsored by the American Society for Bone and Mineral Research. Recognizing the variation in guidelines between multiple national and international societies, the American Society for Bone and Mineral Research developed a multistakeholder international coalition of 35 societies to develop a consensus approach to secondary fracture prevention [10]. The coalition worked out consensus clinical recommendations for management of patients 65 years or older with hip or vertebral fractures. This document describes 13 recommendations covering all aspects of management of these patients, including diagnosis, lifestyle interventions, and fall risk assessment. The document affirms the importance of multidisciplinary care, such as provided by fracture liaison services, to coordinate care, with recommendation to begin pharmacological treatment early after fracture. Oral bisphosphonates may be started within several days of fracture, with intravenous bisphosphonates within 2 weeks after hip fracture, unless contraindicated. The recommendations emphasize communication with patients and providers, education of the patient, and referral back to primary care providers to obtain BMD testing and appropriate clinical follow-up.

These secondary fracture prevention recommendations are consistent with the majority of available national and international guidelines, and support the continuing effort to close the treatment gap in osteoporosis. The strategies promoted targeting the highest risk patients after hip or vertebral fractures, with strong evidence supporting cost- effectiveness of management within multidisciplinary and coordinated models of care, as shown by fracture liaison services. Wider application of these services, in addition to education of patient and health care providers regarding benefits and risk of treatment, gives the best opportunity to reduce the huge burden of secondary fractures in the older population.

Another key initiative, undertaken by the Endocrine Society and endorsed by the European Society of Endocrinology, is the publication of the recent Guideline on the Pharmacologic Management of Postmenopausal Osteoporosis [1]. That document proposes using country-specific fracture risk assessment tools to stratify risk as a starting point for selecting therapy in individual patients. The Guideline emphasizes targeting the highest risk groups of women for the most potent therapies, potentially to longer courses of treatment, and to ongoing risk assessment during treatment interruptions. Those women classified as high or as very high risk are those with a prior spine or hip fracture or those with multiple fractures, respectively, and with appropriately low BMD $\mathrm{T}$ scores. These are exactly the women and men in whom secondary fracture prevention is so sorely needed.

Various approaches may be necessary to overcome barriers limiting universal access to treatment for patients with hip or vertebral and other fractures. Private and governmental funding of the necessary resources, with appropriate reimbursement to maintain excellent clinical care, is needed. Overcoming patient reluctance is also essential, along with greater alignment of specialty care, where the urgency and need for secondary fracture prevention is better appreciated, with primary care. The bridge must be strengthened and the gap crossed to deliver the benefits of secondary fracture prevention to patients at risk for the disability and enhanced mortality of repeated fragility fractures.

Funding This study was supported by Department of Veterans Affairs, Medical Research Service.

\section{Compliance with ethical standards}

Conflict of interest The authors declare that they have no conflict of interest.

Publisher's note Springer Nature remains neutral with regard to jurisdictional claims in published maps and institutional affiliations.

\section{References}

1. R. Eastell, C.J. Rosen, D.M. Black, A.M. Cheung, H.M. Murad, D.M. Shoback, Pharmacological management of osteoporosis in postmenopausal women: an Endocrine Society Clinical Practice Guideline. J. Clin. Endocrinol. Metab. 104, 1595-1622 (2019) 
2. J.E. Compston, M.R. McClung, W.D. Leslie, Osteoporosis. Lancet 393, 364-376 (2019)

3. New Report on Burden of Osteoporosis Highlights Huge and Growing Economic Human Toll of the Disease. (2019). https://www.nof.org/news-events/. Accessed 17 May 2020

4. D.H. Solomon, S.S. Johnston, N.N. Boytsov, D. McMorrow, J.M. Lane, K.D. Krohn, Osteoporosis medication use after hip fracture in U.S. patients between 2002 and 2011. J. Bone Miner. Res. 29, 1929-1937 (2014)

5. S. Khosla, J.A. Cauley, J. Compston et al. Addressing the crisis in the treatment of osteoporosis: a path forward. J. Bone Miner. Res. 32, 424-430 (2017)

6. R.J. Desai, M. Maheshri, Y. Abdia et al. Association of osteoporosis medication use after hip fracture with prevention of subsequent nonvertebral fractures: an instrumental variable analysis. JAMA N. Open 1, e180826 (2018)
7. S. Hawley, J. Leal, A. Delmestri et al. Anti-osteoporosis medication prescriptions and incidence of subsequent fracture among primary hip fracture patients in England and Wales: an interrupted time-series analysis. J. Bone Miner. Res. 31, 2008-2015 (2016)

8. C.H. Wu, S.T. Tu, Y.F. Chang et al. Fracture liaison services improve outcomes of patients with osteoporosis-related fractures: a systematic literature review and meta-analysis. Bone 111, 92-100 (2018)

9. N. Kolanu, A.S. Brown, A. Beech, J. Center, C.P. White, Natural language processing of radiology reports improves identification of patients with fracture. J Endo. Soc. 4, (Suppl 1) (2020), OR2902. https://doi.org/10.1210/jendso/bvaa046.1619

10. R.B. Conley, G. Adib, R.A. Adler et al. Secondary fracture prevention: consensus clinical recommendations from a multistakeholder coalition. J. Bone Miner. Res. 35, 36-52 (2020) 